\title{
Methods for Fault Diagnosability Analysis of a Class of Affine Nonlinear Systems
}

\author{
Xiafu Peng, ${ }^{1}$ Lixiong Lin, ${ }^{1}$ Xunyu Zhong, ${ }^{1}$ and Chengrui Liu ${ }^{2,3}$ \\ ${ }^{1}$ Department of Automation, Xiamen University, Xiamen 361005, China \\ ${ }^{2}$ Science and Technology on Space Intelligent Control Laboratory, Beijing 100190, China \\ ${ }^{3}$ Beijing Institute of Control Engineering, Beijing 100190, China
}

Correspondence should be addressed to Xunyu Zhong; zhongxunyu@xmu.edu.cn

Received 10 December 2014; Accepted 13 January 2015

Academic Editor: Francesco Tornabene

Copyright (C) 2015 Xiafu Peng et al. This is an open access article distributed under the Creative Commons Attribution License, which permits unrestricted use, distribution, and reproduction in any medium, provided the original work is properly cited.

\begin{abstract}
The fault diagnosability analysis for a given model, before developing a diagnosis algorithm, can be used to answer questions like "can the fault $f_{i}$ be detected by observed states?" and "can it separate fault $f_{i}$ from fault $f_{j}$ by observed states?" If not, we should redesign the sensor placement. This paper deals with the problem of the evaluation of detectability and separability for the diagnosability analysis of affine nonlinear system. First, we used differential geometry theory to analyze the nonlinear system and proposed new detectability criterion and separability criterion. Second, the related matrix between the faults and outputs of the system and the fault separable matrix are designed for quantitative fault diagnosability calculation and fault separability calculation, respectively. Finally, we illustrate our approach to exemplify how to analyze diagnosability by a certain nonlinear system example, and the experiment results indicate the effectiveness of the fault evaluation methods.
\end{abstract}

\section{Introduction}

Analyzing the fault diagnosability of system, which is very important for optimizing sensors placement, should be solved firstly before the fault diagnosis. In theory, the more sensors it has the better it is for fault diagnosis and monitoring. However, the sensors placement is constrained by the cost of sensitive component and the installation space, such as the satellite system. Diagnosability assessment is the key to evaluate the performance that one can expect from a diagnoser at run time and to define the appropriate set of sensors to be included in the design of a system $[1,2]$.

The model-based approach to fault detection and isolation (FDI) has received a lot of attention. At first, the focus is on the linear system. Based on the transfer function method [3-5], the transfer function between residual and the fault is obtained. Through the transfer function, the diagnosability of faults is evaluated. In [6], the fault is detectable if the transfer function is not equal to zero. At the same time, the transfer function between residual and disturbance is used to determine whether the disturbance can be decoupled or not
[7]. In the case of the above conditions, the fault is regarded as separable, and then a set of unknown input observers are designed to realize fault isolation [8]. The FDI of nonlinear system is researched in $[9,10]$, and the FDI of uncertain linear systems is also concerned [11, 12]. However, compared with the rich results in FDI of linear and nonlinear systems, only a limited number of contributions about fault diagnosability analysis have been found, especially for the nonlinear system. In the field of linear continuous systems, diagnosability is formulated in terms of fault detectability and isolability which provides a survey of definitions from different points of view [13-15], and diagnosability which includes detectability and distinguishability are analyzed for discrete-event systems using labeled Petri net [16].

To the best of the authors' knowledge, up to now, almost no attention has been paid to the study of fault detectability criterion and fault separability criterion for nonlinear systems with faults occurring, though the diagnosability analysis and judgment of linear continuous system are focused [17]. But we notice that the differential geometry based method is one of the most important methods in robust fault diagnosis of 
nonlinear system [18-21]. The problem of fault diagnosis is converted into residual generator problem [18]. A solution to the problem of fault detection and isolation is characterized in terms of properties of certain distributions, which can be considered as the nonlinear analog of the unobservability subspaces [19]. The same problem is solved and the sufficient and necessary conditions are given by some distributions named unobservability codistribution [20]. And the appropriate coordinate transformation based on the principle of differential geometry can be designed to make one subsystem only affected by one fault, and then the fault is detectable and separable.

In this paper, the methods, based on the principle of differential geometry, are proposed to analyze the fault detectability and separability (FDS) different to FDI. Usually, the attention is on the approach to FDI, and the conditions that make the faults be diagnosable are given based on the designed approaches. However, in this paper, the diagnosability of fault is regarded as the inherent attribute of the system and is not changed with the approach of diagnosis.

Affine nonlinear system is the most common system, such as induction/synchronous motor, rigid robot, space craft, and satellite. The main contributions of this paper are summarized as follows. For a class of affine nonlinear systems with faults occurring, (1) the definitions of detectability and separability are given and the criteria to judge detectability and separability are proposed and proved and (2) the detectability incidence matrix and separability incidence matrix are defined for quantitative fault diagnosability calculation and fault separability calculation, respectively.

\section{System Model and Basic Properties}

Consider the following affine nonlinear system:

$$
\begin{aligned}
& \dot{x}=f(x)+\sum_{i=1}^{m} g_{i}(x) u_{i}, \\
& y=\left[\begin{array}{lll}
h_{1}(x) & \cdots & h_{l}(x)
\end{array}\right],
\end{aligned}
$$

where $x \in \mathbf{R}^{n}$ is the state vector, $u_{i}$ is the input $(i=$ $1,2, \ldots, m)$, and $y \in \mathbf{R}^{l}$ is the output vector.

According to the principle of differential geometry, let us introduce the following lemmas which will be used in deriving our main results.

Lemma 1 (output invariance). Given the affine nonlinear model (1), output $y_{j}=h_{j}(x)$ will be not affected by $u_{i}$ (i.e., the output is invariant to the input), if and only if it has distribution $\Delta$ and satisfies the following conditions:

(1) distribution $\Delta$ is invariant under vector field $\left\{f, g_{1}, g_{2}, \ldots, g_{m}\right\}$

(2) vector field $g_{i} \in \Delta \subset\left(\operatorname{span}\left\{d h_{j}\right\}\right)^{\perp}$.

Lemma 2 (transformation of coordinates). Given the affine nonlinear model (1), if its distribution $\left\langle f, g_{1}, \ldots, g_{m}\right|$ $\left.\operatorname{span}\left\{d h_{1}, \ldots, d h_{l}\right\}\right\rangle^{\perp}$ is $d$-dimensional and nonsingular, then $\forall x_{0}$ of the domain one can find a neighborhood $U_{0}$ and the transformation of local coordinates $\xi=\left(\xi_{1}, \xi_{2}\right)=\Phi(x)$ defined in $U_{0}$. In the new coordinate system, (1) can be denoted by

$$
\begin{gathered}
\dot{\xi}_{1}=\eta_{1}(\xi)+\sum_{i=1}^{m} \psi_{1 i}(\xi) u_{i}, \\
\dot{\xi}_{2}=\eta_{2}\left(\xi_{2}\right)+\sum_{i=1}^{m} \psi_{2 i}\left(\xi_{2}\right) u_{i}, \\
y=\left[\lambda_{1}\left(\xi_{2}\right), \ldots, \lambda_{l}\left(\xi_{2}\right)\right],
\end{gathered}
$$

where $\xi_{1}$ is d-dimensional.

\section{Fault Diagnosability Analysis}

In the affine nonlinear system, its faults are regarded as the inputs of the system. The fault diagnosability evaluation includes two aspects: faults detection and faults separation.

3.1. Fault Detectability Judgment. Consider the following affine nonlinear system:

$$
\begin{gathered}
\dot{x}=f(x)+\sum_{i=1}^{m} g_{i}(x) u_{i}+\sum_{i=1}^{s} p_{i}(x) w_{i}, \\
y=\left[\begin{array}{lll}
h_{1}(x) & \cdots & h_{l}(x)
\end{array}\right]
\end{gathered}
$$

where $w_{i}$ denotes the fault and $p_{i}(x)$ denotes its coupled mode $(i=1,2, \ldots, s)$.

Definition 3 (detectability). Given the affine nonlinear model (3), if outputs $\mathbf{y}$ will be not influenced by $w_{i}$ (i.e., y is invariant to $w_{i}$ ), then fault $w_{i}$ will be not detected by $\mathbf{y}$; that is, $w_{i}$ is undetectable; otherwise, $w_{i}$ will be detected by $\mathbf{y}$; that is, $w_{i}$ is detectable. The fact that the ability of faults will be detected by output is named as detectability.

And then, we propose the following proposition as the criteria to judge detectability.

Proposition 4 (detectability judgment). Given the affine nonlinear model (3), the necessary and sufficient condition for $w_{i}$ being detectable is

$$
p_{i} \notin\left\langle f, g_{i}, \ldots, g_{m}, p_{1}, \ldots, p_{s} \mid \operatorname{span}\left\{d h_{1}, \ldots, d h_{l}\right\}\right\rangle^{\perp},
$$

where $\left\langle f, g_{i}, \ldots, g_{m}, p_{1}, \ldots, p_{s} \quad \mid \quad \operatorname{span}\left\{d h_{1}, \ldots, d h_{l}\right\}\right\rangle^{\perp}$ is the maximal distribution which is contained in $\left(\operatorname{span}\left\{d h_{1}, \ldots, d h_{l}\right\}\right)^{\perp}$ and is invariant under the vector field $\left\{f, g_{i}, \ldots, g_{m}, p_{1}, \ldots, p_{s}\right\}$.

Proof. (1) The necessary condition, that is, if $p_{i} \notin\left\langle f, g_{i}, \ldots\right.$, $g_{m}, p_{1}, \ldots, p_{s}\left|\operatorname{span}\left\{d h_{1}, \ldots, d h_{l}\right\}\right\rangle^{\perp}$, then it has that $w_{i}$ is detectable.

Make a proof by contradiction, supposing that $w_{i}$ is not detectable when

$$
p_{i} \notin\left\langle f, g_{i}, \ldots, g_{m}, p_{1}, \ldots, p_{s} \mid \operatorname{span}\left\{d h_{1}, \ldots, d h_{l}\right\}\right\rangle^{\perp}
$$

then $\mathbf{y}$ will be not influenced by $w_{i}$ according to Definition 3, and $\forall d h_{j} \in\left\{d h_{1}, \ldots, d h_{l}\right\}$, it has 
$p_{i} \in\left\langle f, g_{i}, \ldots, g_{m}, p_{1}, \ldots, p_{s} \quad \mid \operatorname{span}\left\{d h_{j}\right\}\right\rangle^{\perp}$ according to Lemma 1; that is,

$$
\begin{aligned}
p_{i} \in( & \left\langle f, g_{i}, \ldots, g_{m}, p_{1}, \ldots, p_{s} \mid \operatorname{span}\left\{d h_{1}\right\}\right\rangle^{\perp} \\
& \left.\cap \cdots \cap\left\langle f, g_{i}, \ldots, g_{m}, p_{1}, \ldots, p_{s} \mid \operatorname{span}\left\{d h_{l}\right\}\right\rangle^{\perp}\right) .
\end{aligned}
$$

According to the distribution algorithm $\Delta_{1}{ }^{\perp} \cap \Delta_{2}{ }^{\perp}=\left(\Delta_{1} U\right.$ $\left.\Delta_{2}\right)^{\perp}$ (where $\Delta_{1}$ and $\Delta_{2}$ are any distribution), we get the conclusion that

$$
\begin{aligned}
p_{i} \in\left\langle f, g_{i}, \ldots, g_{m}, p_{1}, \ldots, p_{s}\right| \\
\left.\quad \operatorname{span}\left\{d h_{1}\right\} \cup \cdots \cup \operatorname{span}\left\{d h_{l}\right\}\right\rangle^{\perp} ;
\end{aligned}
$$

that is,

$$
p_{i} \in\left\langle f, g_{i}, \ldots, g_{m}, p_{1}, \ldots, p_{s} \mid \operatorname{span}\left\{d h_{1}, \ldots, d h_{l}\right\}\right\rangle^{\perp} .
$$

This is contradictory with the supposed equation (5). So, the necessary condition is true.

(2) The sufficient condition, that is, if $w_{i}$ is detectable, then it has that

$$
p_{i} \notin\left\langle f, g_{i}, \ldots, g_{m}, p_{1}, \ldots, p_{s} \mid \operatorname{span}\left\{d h_{1}, \ldots, d h_{l}\right\}\right\rangle^{\perp} .
$$

Make a proof by contradiction, supposing that when $w_{i}$ is detectable, it can get the conclusion that

$$
p_{i} \in\left\langle f, g_{i}, \ldots, g_{m}, p_{1}, \ldots, p_{s} \mid \operatorname{span}\left\{d h_{1}, \ldots, d h_{l}\right\}\right\rangle^{\perp}
$$

then, according to the distribution algorithm $\left(\Delta_{1} \cup \Delta_{2}\right)^{\perp}=$ $\Delta_{1}{ }^{\perp} \cap \Delta_{2}{ }^{\perp}, \forall d h_{j} \in\left\{d h_{1}, \ldots, d h_{l}\right\}$, it has

$$
p_{i} \in\left\langle f, g_{i}, \ldots, g_{m}, p_{1}, \ldots, p_{s} \mid \operatorname{span}\left\{d h_{j}\right\}\right\rangle^{\perp} .
$$

So, according to Lemma 1 , for all $j=1, \ldots, l, y_{j}=h_{j}(x)$ (where $h_{j} \in\left\{h_{1}, \ldots, h_{l}\right\}$ ) will be not influenced by $w_{i}$; that is, $w_{i}$ is not detectable, which is contradictory with the supposing that $w_{i}$ is detectable. So, the sufficient condition is true.

This completes the proof of Proposition 4.

3.2. Evaluation Indicator of the Detectability. Here, we design the incidence matrix and quantitative fault diagnosability calculation method as the following definitions.

Definition 5 (detectability incidence matrix). For detectability incidence matrix $\Pi_{l * s}$, its element is denoted by $\pi_{j i}(1 \leq$ $j \leq l, 1 \leq i \leq s$ ). If $w_{i}$ will be detected by $y_{j}=h_{j}(x)$ (i.e., $w_{i}$ is detectable), then set the value of $\pi_{j i}$ as 1 ; else if $w_{i}$ will be not detected by $y_{j}=h_{j}(x)$ (i.e., $w_{i}$ is not detectable), then set the value of $\pi_{j i}$ as 0 .

Definition 6 (diagnosability calculation). DFD (Degree of Fault Detectability) is as follows:

$$
\mathrm{DFD}=\frac{n}{s} \times 100 \%,
$$

where $n$ is the number of the nonzero column vectors in $\Pi_{l * s}$ and $s$ is the sum of the faults.
3.3. Fault Separability Judgment. First, we give the definition of faults separability. And then, we propose the following proposition as the criteria to judge separability.

Definition 7 (separability). For the detectable faults $w_{i}$ and $w_{j}$ of nonlinear system (3), there is the ability to separate $w_{i}$ from $w_{j}$ when the influence of outputs $\mathbf{y}$ affected by $w_{i}$ is different from $w_{j}$, and this ability is named as separability.

For the detectable faults, we can judge their separability by the distribution (space) spanned by the fault vector field (i.e., the coupled mode of fault).

(1) If the spanned distributions $\operatorname{span}\left(p_{i}\right)$ and $\operatorname{span}\left(p_{j}\right)$ are equivalent, then the influence of $w_{i}$ on the outputs $y$ is same as the influence of $w_{j}$; that is, $w_{i}$ and $w_{j}$ are inseparable. Otherwise, $w_{i}$ and $w_{j}$ are separable.

(2) When the distributions ( $\operatorname{space}) \operatorname{span}\left(p_{i}\right)$ and $\operatorname{span}\left(p_{j}\right)$ are not equivalent, the following proposition will be used to judge the separability of $w_{i}$ and $w_{j}$.

Proposition 8 (separability judgment). Given the affine nonlinear system (3) and its detectable faults $w_{i}$ and $w_{j}$ (fault vector fields are $p_{i}$ and $\left.p_{j}\right)$, if $\operatorname{span}\left\{p_{i}\right\} \neq \operatorname{span}\left\{p_{j}\right\}$, then $w_{i}$ and $w_{j}$ are inseparable if all $p$ (where $p \in \Delta, \Delta=\operatorname{span}\left\{p_{i}\right\} \cup$ $\left.\operatorname{span}\left\{p_{j}\right\}-\operatorname{span}\left\{p_{i}\right\} \cap \operatorname{span}\left\{p_{j}\right\}\right)$ satisfy

$$
p \in\left\langle f, g_{i}, \ldots, g_{m}, p_{1}, \ldots, p_{s} \mid \operatorname{span}\left\{d h_{1}, \ldots, d h_{l}\right\}\right\rangle^{\perp} .
$$

Otherwise, $w_{i}$ and $w_{j}$ are separable.

Proof. (1) The sufficient condition (proof by contradiction) is as follows. Let

$$
\Delta=\operatorname{span}\left\{p_{i}\right\} \cup \operatorname{span}\left\{p_{j}\right\}-\operatorname{span}\left\{p_{i}\right\} \cap \operatorname{span}\left\{p_{j}\right\} .
$$

all $p($ where $p \in \Delta)$ satisfy

$$
p \notin\left\langle f, g_{i}, \ldots, g_{m}, p_{1}, \ldots, p_{s} \mid \operatorname{span}\left\{d h_{1}, \ldots, d h_{l}\right\}\right\rangle^{\perp}
$$

then the different influence on outputs $\mathbf{y}$ affected by $w_{i}$ and $w_{j}$ will be detected according to Lemma 1, so we can know that the faults $w_{i}$ and $w_{j}$ are separable according to Definition 7.

(2) The necessary condition (proof by contradiction) is as follows. According to Definition 7 , if the detectable faults $w_{i}$ and $w_{j}$ are separable, then the influences on outputs $\mathbf{y}$ affected by $w_{i}$ and $w_{j}$ are different and detectable. Since the distribution $\Delta=\operatorname{span}\left\{p_{i}\right\} \cup \operatorname{span}\left\{p_{j}\right\}-\operatorname{span}\left\{p_{i}\right\} \cap \operatorname{span}\left\{p_{j}\right\}$ represents the different influences by $w_{i}$ and $w_{j}$, according to Lemma 1 , we can know that all $p$ (where $p \in \Delta$ ) satisfy

$$
p \notin\left\langle f, g_{i}, \ldots, g_{m}, p_{1}, \ldots, p_{s} \mid \operatorname{span}\left\{d h_{1}, \ldots, d h_{l}\right\}\right\rangle^{\perp} .
$$

It is contradictory with (13). So, the fact that the faults $w_{i}$ and $w_{j}$ are separable is true.

This completes the proof of Proposition 8.

3.4. Evaluation Indicator of Separability. Similarly, we design the incidence matrix and quantitative fault separability calculation method as the following definitions. 
Definition 9 (separability incidence matrix). For separability incidence matrix $\Gamma_{s * *}$, its element is denoted by $\Gamma_{i j}(1 \leq i \leq s$, $1 \leq j \leq s) . \Gamma_{i j}(i \neq j)$ is to express the separability of $w_{i}$ and $w_{j}$. If $w_{i}$ and $w_{j}$ are separable, then set the value of $\Gamma_{i j}$ as 1 or else the value as 0 ; and set $\Gamma_{i i}=0$ particularly.

Definition 10 (separability calculation). DFS (Degree of Fault Separation) is as follows:

$$
\mathrm{DFS}=\frac{\sum_{j=1}^{s} \sum_{i=1}^{s} \Gamma_{i j}}{s(s-1)} \times 100 \%,
$$

where $\Gamma_{i j}$ is the element of $\Gamma_{s * s}$ and $s$ is the sum of the detectable faults.

\section{Illustration Example}

The following affine nonlinear system is given as an example to demonstrate the fault diagnosability analysis methods outlined above:

$$
\begin{gathered}
\dot{x_{1}}=x_{1} x_{2}+x_{3} e^{x_{3}}+x_{1} u+x_{1} w_{2}, \\
\dot{x_{2}}=-x_{2} x_{3}+w_{1}+w_{3}, \\
\dot{x_{3}}=x_{2}+u+x_{2} w_{1}, \\
\dot{x_{4}}=x_{2}^{2}+x_{3} x_{4}-x_{2} x_{3}^{2}+x_{2} u, \\
y=x_{3},
\end{gathered}
$$

where $w_{1}, w_{2}$, and $w_{3}$ are the faults of the system and

$$
\begin{gathered}
f(x)=\left[\begin{array}{c}
x_{1} x_{2}+x_{3} e^{x_{3}} \\
-x_{2} x_{3} \\
x_{2} \\
x_{2}{ }^{2}+x_{3} x_{4}-x_{2} x_{3}{ }^{2}
\end{array}\right], \\
g(x)=\left[\begin{array}{c}
x_{1} \\
0 \\
1 \\
x_{2}
\end{array}\right], \quad p_{1}(x)=\left[\begin{array}{l}
0 \\
1 \\
x_{2} \\
0
\end{array}\right], \\
p_{2}(x)=\left[\begin{array}{l}
x_{1} \\
0 \\
0 \\
0
\end{array}\right], \quad p_{3}(x)=\left[\begin{array}{l}
0 \\
1 \\
0 \\
0
\end{array}\right] .
\end{gathered}
$$

\subsection{Diagnosability Analysis}

4.1.1. Analyze the Detectability of the Faults. After calculation, we obtain

$$
\begin{aligned}
& p_{1} \notin\left\langle f, g, p_{1}, p_{2} \mid \operatorname{span}\{d h\}\right\rangle^{\perp} \\
& p_{2} \in\left\langle f, g, p_{1}, p_{2} \mid \operatorname{span}\{d h\}\right\rangle^{\perp} \\
& p_{3} \notin\left\langle f, g, p_{1}, p_{2} \mid \operatorname{span}\{d h\}\right\rangle^{\perp} .
\end{aligned}
$$

So, according to Proposition $4, w_{1}$ is detectable, $w_{2}$ is not detectable, and $w_{3}$ is detectable.

Then, the detectability incidence matrix

$$
\Pi_{l * s}=\left[\begin{array}{lll}
1 & 0 & 1
\end{array}\right] .
$$

So, the DFD is

$$
\mathrm{DFD}=\frac{n}{s} \times 100 \%=\frac{2}{3} \times 100 \%=66.7 \% .
$$

4.1.2. Analyze the Separability of the Faults. Here, after calculation, we have $\operatorname{span}\left\{p_{1}\right\} \neq \operatorname{span}\left\{p_{3}\right\}$ and all $p$ (where $p \in \Delta$ ) have

$$
p \notin\left\langle f, g, p_{1}, p_{2}, p_{3} \mid \operatorname{span}\left\{d h_{1}\right\}\right\rangle^{\perp},
$$

where $\Delta=\operatorname{span}\left\{p_{1}\right\} \cup \operatorname{span}\left\{p_{3}\right\}-\operatorname{span}\left\{p_{1}\right\} \cap \operatorname{span}\left\{p_{3}\right\}$. So, $w_{1}$ and $w_{3}$ are separable according to Proposition 8 . The separability incidence matrix

$$
\Gamma_{2 * 2}=\left[\begin{array}{ll}
0 & 1 \\
1 & 0
\end{array}\right] .
$$

So, the DFS of $w_{1}$ and $w_{3}$ is

$$
\text { DFS }=\frac{\sum_{j=1}^{s} \sum_{i=1}^{s} \Gamma_{i j}}{s(s-1)} \times 100 \%=100 \% .
$$

4.2. Diagnosability Verification. According to Lemma 2, it can provide the transformation of local coordinates $\xi=$ $\left(\xi_{1}, \xi_{2}\right)=\phi(x)$ by $\operatorname{span}\left\{d x_{1}, d x_{4}\right\}$, where $\xi_{1}=\left(\begin{array}{ll}\xi_{11} & \xi_{12}\end{array}\right)=$ $\left(\begin{array}{ll}x_{1} & x_{4}\end{array}\right)$ and $\xi_{2}=\left(\begin{array}{ll}\xi_{21} & \xi_{22}\end{array}\right)=\left(\begin{array}{ll}x_{3} & x_{2}\end{array}\right)$. Then, we get the following subsystem equations under the new coordinate system:

$$
\begin{gathered}
\dot{\xi_{21}}=\xi_{22}+u+\xi_{22} w_{1} \\
\dot{\xi_{22}}=-\xi_{21} \xi_{22}+w_{1}+w_{3} \\
y=\xi_{21} .
\end{gathered}
$$

Next, we design the state observer and get the residual error to verify the detectability and separability of the faults.

Let

$$
\begin{gathered}
\varepsilon_{1}=\frac{1}{2} \xi_{21}{ }^{2}+\xi_{22} \\
\varepsilon_{2}=\xi_{21},
\end{gathered}
$$

and then subsystem (26) changed as

$$
\begin{aligned}
\left(\begin{array}{c}
\dot{\varepsilon}_{1} \\
\dot{\varepsilon}_{2}
\end{array}\right)= & A\left(\begin{array}{c}
\varepsilon_{1} \\
\varepsilon_{2}
\end{array}\right)+\left(\begin{array}{c}
0 \\
-\frac{1}{2} \varepsilon_{2}^{2}
\end{array}\right)+\left(\begin{array}{c}
\varepsilon_{2} \\
1
\end{array}\right) u \\
& +\left(\begin{array}{c}
1+\varepsilon_{1} \varepsilon_{2}-\frac{1}{2} \varepsilon_{2}^{3} \\
\varepsilon_{1}-\frac{1}{2} \varepsilon_{2}^{2}
\end{array}\right) w_{1}+\left(\begin{array}{l}
1 \\
0
\end{array}\right) w_{3}, \\
y & =C\left(\begin{array}{l}
\varepsilon_{1} \\
\varepsilon_{2}
\end{array}\right)=\left[\begin{array}{ll}
0 & 1
\end{array}\right]\left(\begin{array}{l}
\varepsilon_{1} \\
\varepsilon_{2}
\end{array}\right)=\varepsilon_{2},
\end{aligned}
$$




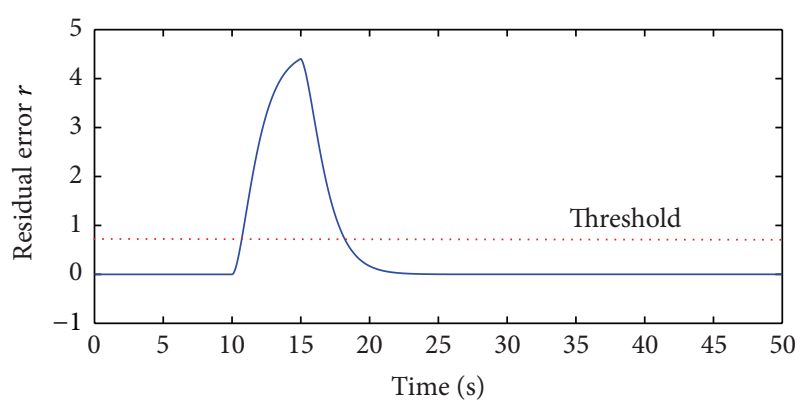

FIGURE 1: The residual error when giving step fault $w_{1}$ at time $10 \sim 15 \mathrm{~s}$.

where

$$
A=\left[\begin{array}{ll}
0 & 0 \\
1 & 0
\end{array}\right], \quad C=\left[\begin{array}{ll}
0 & 1
\end{array}\right] .
$$

Design the state observer as follows:

$$
\dot{\vec{\varepsilon}}=(A+G C)\left(\begin{array}{l}
\widehat{\varepsilon_{1}} \\
\widehat{\varepsilon_{2}}
\end{array}\right)-G y+\left(\begin{array}{c}
0 \\
-\frac{1}{2} y^{2}
\end{array}\right)+\left(\begin{array}{l}
y \\
1
\end{array}\right) u,
$$

where $(A+G C)$ is Hurwitz; here, set $G=\left[\begin{array}{ll}-1 & -2\end{array}\right]^{T}$.

Let

$$
\begin{gathered}
e=\varepsilon-\widehat{\varepsilon}, \\
r=y-\varepsilon_{2},
\end{gathered}
$$

and then

$$
\begin{gathered}
\dot{e}=(A+G C) e+P_{1} w_{1}+P_{3} w_{3}, \\
r=e_{2},
\end{gathered}
$$

where

$$
P_{1}=\left(\begin{array}{c}
1+\varepsilon_{1} y-\frac{1}{2} y^{3} \\
\varepsilon_{1}-\frac{1}{2} y^{2}
\end{array}\right), \quad P_{3}=\left(\begin{array}{l}
1 \\
0
\end{array}\right) .
$$

The residual error output is shown in Figure 1 when giving step fault input $w_{1}$ at time 10 15 s into the system; and when giving step fault input $w_{3}$ at time $10 \sim 15 \mathrm{~s}$, the residual error output is shown in Figure 2. In Figures 1 and 2, we can see that the residual error obviously changed at time 10 15s. Therefore, the faults $w_{1}$ and $w_{3}$ are detectable, same as the above analyses of the detectability.

The residual error output is shown in Figure 3 when giving step fault input $w_{2}$ at time 10 15 s into the system; from Figure 3 , we can see that fault $w_{2}$ is not detectable, same as the above analyses of the detectability.

When giving step fault input $w_{1}$ at time 10 15 s and step fault input $w_{3}$ at time $20 \sim 30 \mathrm{~s}$, the residual error output is shown in Figure 4. In Figure 4, we can see that the residual error obviously changed at time 10 15 s and time 20 30 s separately. Therefore, the faults $w_{1}$ and $w_{3}$ are separable, same as the above analyses of the separability.

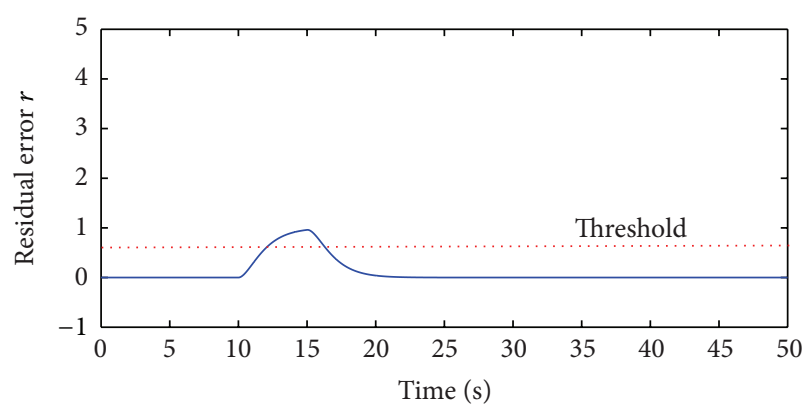

Figure 2: The residual error when giving step fault $w_{3}$ at time $10 \sim 15 \mathrm{~s}$.

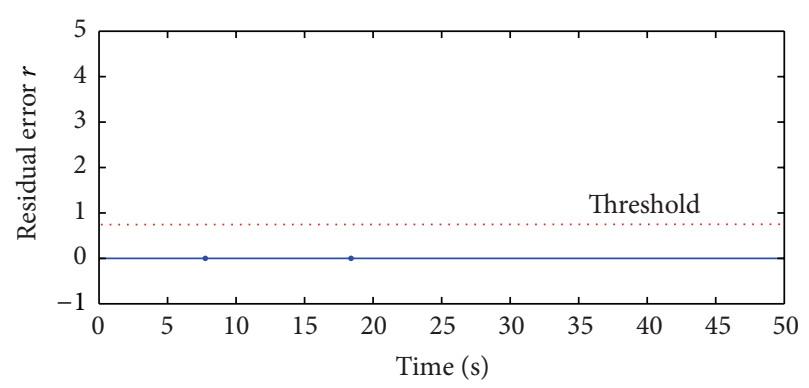

FIGURE 3: The residual error when giving step fault $w_{2}$ at time 10 $15 \mathrm{~s}$.

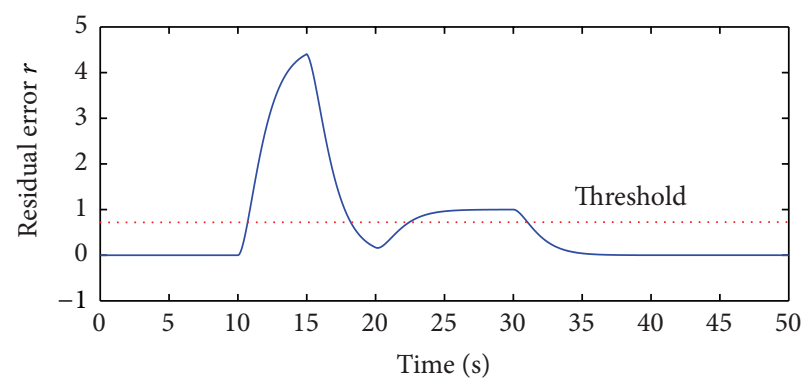

FIGURE 4: The residual error when giving step fault $w_{1}$ at time $10 \sim 15 \mathrm{~s}$ and step fault $w_{3}$ at time 20 30 s.

\section{Conclusion}

In this paper, we use the principle of differential geometry to analyze the problem of diagnosability of a class of affine nonlinear systems with faults. We first give the necessary and sufficient conditions for (1) detectability judgment, that is, the conditions which the system should satisfy whether the faults are detectable or not, and (2) separability judgment, that is, the conditions which the system should satisfy whether the faults are separable or not. And the measurement, that is, DFD (Degree of Fault Detectability) and DFS (Degree of Fault Separation), based on the defined DIM (detectability incidence matrix) and SIM (separability incidence matrix) is established to quantify diagnosability performance. Then, we have provided an example to analyze and test the faults diagnosability. However, the simple quantitative measure indexes, DFD and DFS, have not taken full advantage of the full information of DIM and SIM. More comprehensive 
quantitative measure index will be designed in our future research.

\section{Conflict of Interests}

The authors declare that there is no conflict of interests regarding the publication of this paper.

\section{Acknowledgments}

This work was supported by the National Natural Science Foundation of China (Grant no. 61305117), 973 subproject, and National Key Laboratory Foundation of China (Grant no. 9140c59030411ht05). The authors are grateful to the editors and the reviewers for their valuable comments and suggestions that greatly helped to improve the quality of this paper.

\section{References}

[1] E. Frisk, M. Krysander, and J. Aslund, "Sensor placement for fault isolation in linear differential-algebraic systems," Automatica, vol. 45, no. 2, pp. 364-371, 2009.

[2] M. Bayoudh and L. Travé-Massuyès, "Diagnosability analysis of hybrid systems cast in a discrete-event framework," Discrete Event Dynamic Systems: Theory and Applications, vol. 24, no. 3, pp. 309-338, 2014.

[3] P. M. Frank and X. Ding, "Survey of robust residual generation and evaluation methods in observer-based fault detection systems," Journal of Process Control, vol. 7, no. 6, pp. 403-424, 1997.

[4] C. G. Siontorou, F. A. Batzias, and V. Tsakiri, "A knowledgebased approach to online fault diagnosis of FET biosensors," IEEE Transactions on Instrumentation and Measurement, vol. 59, no. 9, pp. 2345-2364, 2010.

[5] A. P. Moreno, O. L. Santiago, J. M. B. de Lázaro, and E. G. Moreno, "Comparative evaluation of classification methods used in fault diagnosis of industrial processes," IEEE Latin America Transactions, vol. 11, no. 2, pp. 682-689, 2013.

[6] F. Li, B. R. Upadhyaya, and S. R. P. Perillo, "Fault diagnosis of helical coil steam generator systems of an integral pressurized water reactor using optimal sensor selection," IEEE Transactions on Nuclear Science, vol. 59, no. 2, pp. 403-410, 2012.

[7] Y. Shen, "Fault diagnosis based on high-gain observer with an update law for a class of nonlinear systems," Journal of Control Theory and Applications, vol. 8, no. 4, pp. 447-452, 2010.

[8] H. Hamdi, M. Rodrigues, C. Mechmeche, and N. B. Braiek, "Robust fault detection and estimation for descriptor systems based on multi-models concept," International Journal of Control, Automation and Systems, vol. 10, no. 6, pp. 1260-1266, 2012.

[9] M. Shahriari-Kahkeshi and F. Sheikholeslam, "Adaptive fuzzy wavelet network for robust fault detection and diagnosis in nonlinear systems," IET Control Theory \& Applications, vol. 8, no. 15, pp. 1487-1498, 2014.

[10] V. Reppa, M. M. Polycarpou, and C. G. Panayiotou, "Adaptive approximation for multiple sensor fault detection and isolation of nonlinear uncertain systems," IEEE Transactions on Neural Networks and Learning Systems, vol. 25, no. 1, pp. 137-153, 2014.

[11] Q. Su and J. Li, "Fault detection for a class of uncertain linear systems," Mathematical Problems in Engineering, vol. 2013, Article ID 856914, 12 pages, 2013.
[12] X.-J. Li and G.-H. Yang, "Robust fault detection and isolation for a class of uncertain single output non-linear systems," IET Control Theory and Applications, vol. 8, no. 7, pp. 462-470, 2014.

[13] M. Basseville, M. Kinnaert, and M. Nyberg, "On fault detectability and isolability," European Journal of Control, vol. 7, no. 6, pp. 625-637, 2001.

[14] D. Eriksson, E. Frisk, and M. Krysander, "A method for quantitative fault diagnosability analysis of stochastic linear descriptor models," Automatica, vol. 49, no. 6, pp. 1591-1600, 2013.

[15] Y. Cui, J. Shi, and Z. Wang, "System-level operational diagnosability analysis in quasi real-time fault diagnosis: the probabilistic approach," Journal of Process Control, vol. 24, no. 9, pp. 14441453, 2014.

[16] M. P. Cabasino, A. Giua, and C. Seatzu, "Diagnosability of discrete-event systems using labeled petri nets," IEEE Transactions on Automation Science and Engineering, vol. 11, no. 1, pp. 144-153, 2014.

[17] W. Liu, C. Liu, N. Wang, and D. Wang, "Quantitative and qualitative model based fault diagnosability evaluation of momentum wheel," Chinese Space Science and Technology, vol. 31, no. 4, pp. 54-62, 2011.

[18] C. Verde, "Robust fault detection for nonlinear hessenberg systems," in Proceedings of the American Control Conference, pp. 3287-3292, Anchorage, Alaska, USA, May 2002.

[19] C. de Persis and A. Isidori, "A geometric approach to nonlinear fault detection and isolation," IEEE Transactions on Automatic Control, vol. 46, no. 6, pp. 853-865, 2001.

[20] X. Zhang, X. Peng, and X. Zhong, "Nonlinear fault detection and isolation via unobservability codistribution," in Proceedings of the IEEE Conference on Prognostics and System Health Management (PHM'12), pp. 1-6, Beijing, China, May 2012.

[21] P. Castaldi, N. Mimmo, and S. Simani, "Differential geometry based active fault tolerant control for aircraft," Control Engineering Practice, vol. 32, pp. 227-235, 2014. 


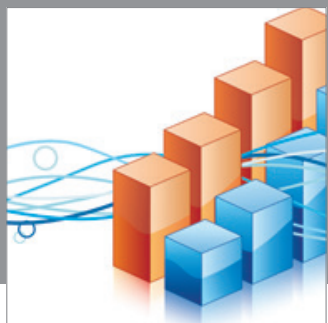

Advances in

Operations Research

mansans

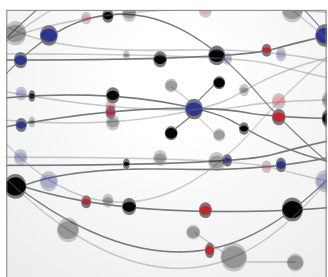

The Scientific World Journal
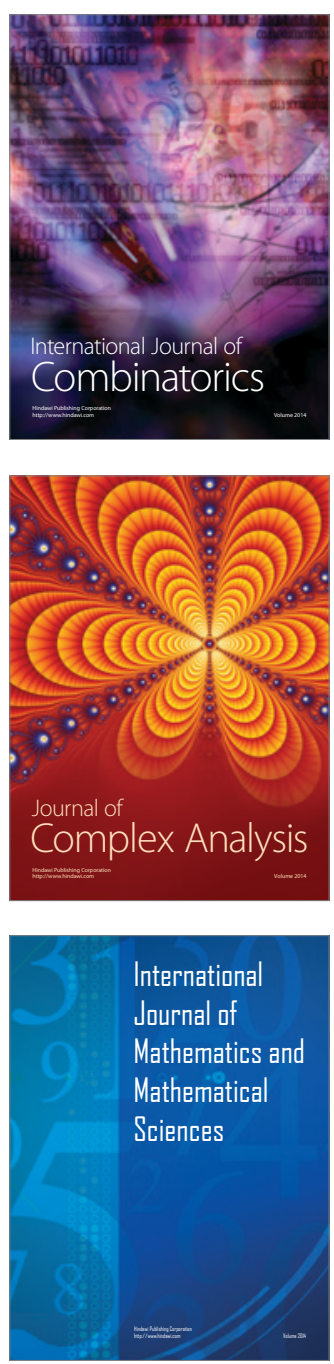
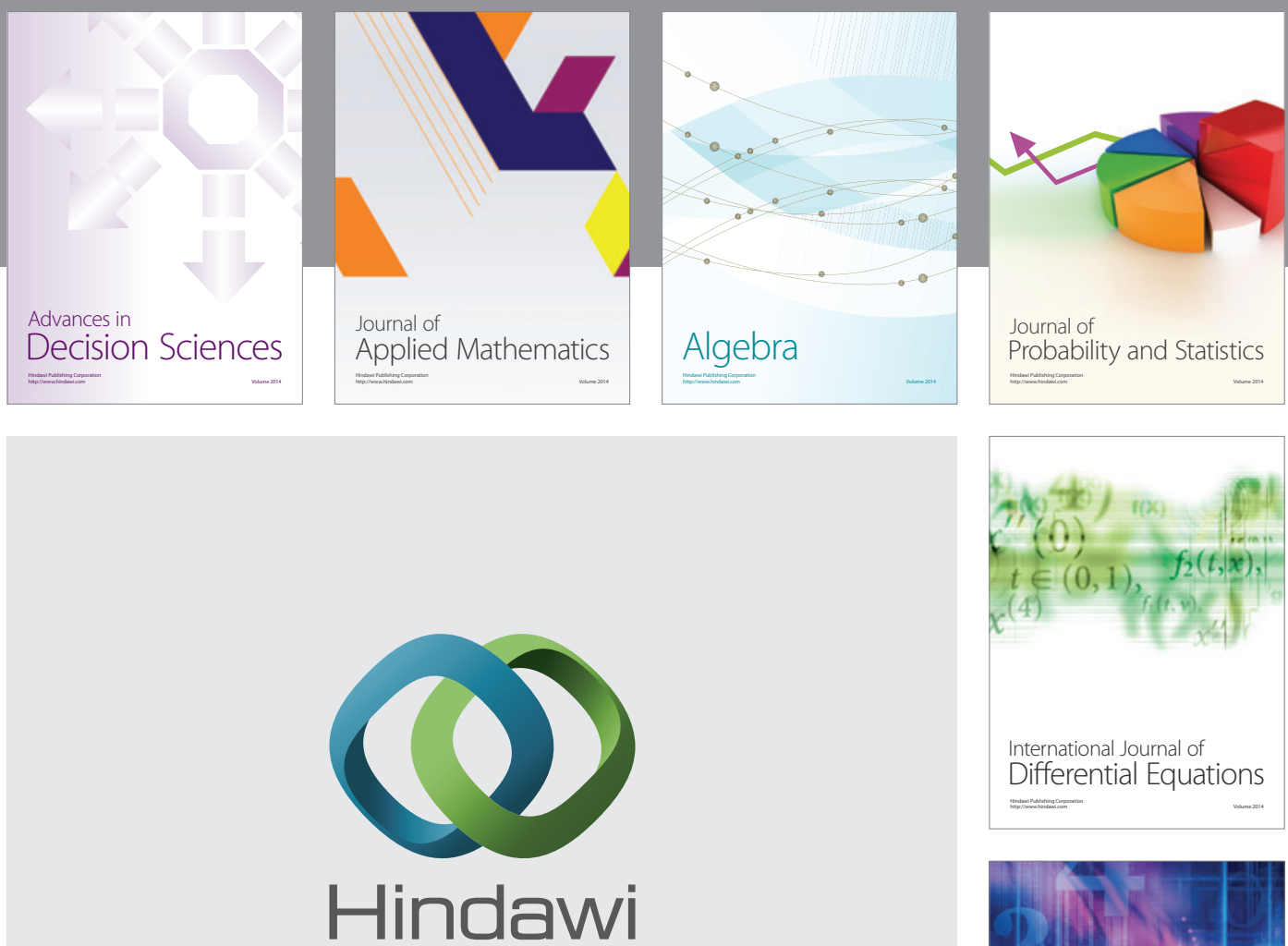

Submit your manuscripts at http://www.hindawi.com
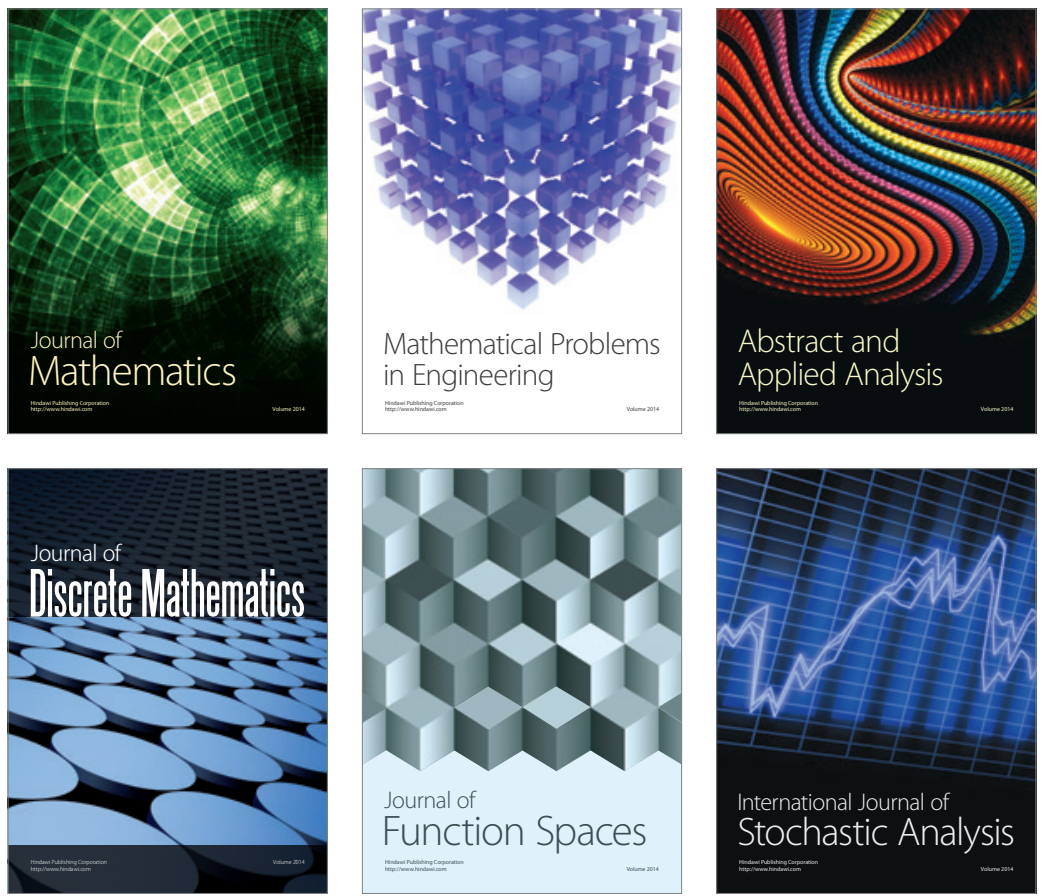

Journal of

Function Spaces

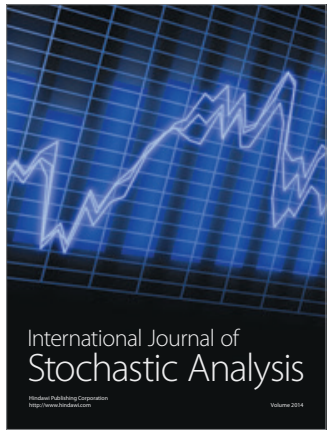

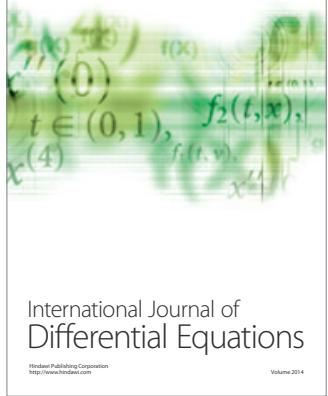
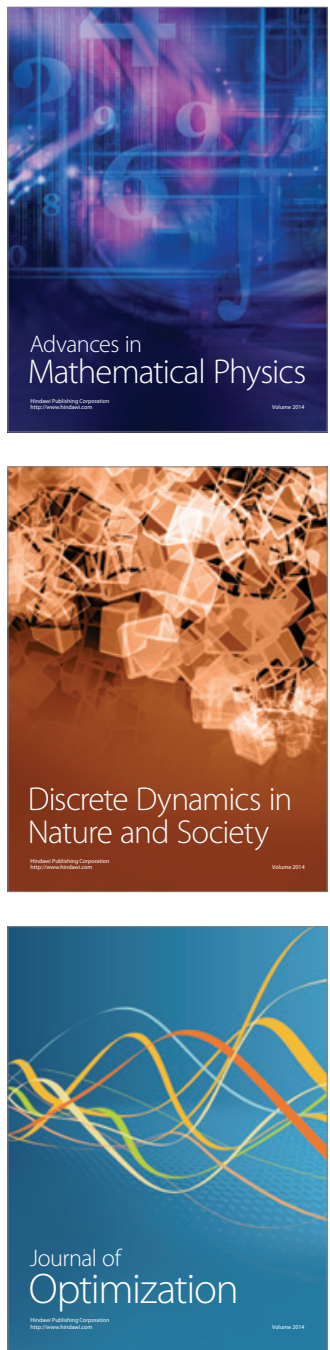